\title{
WHY DO INSECTS BITE? A review of blood sucking behaviour
}

\author{
CS Dodd \\ $\mathrm{NRH}$ Burgess
}

Department of Military Entomology, Royal Army Medical College, Millbank, London SW1P 4RJ

SUMMARY: This paper answers the question 'Why do insects bite?' by examining the sequence in which the insect receives the stimuli that enable it to find a host, and subsequent portioning of the blood meal once the insect has fed.

\section{Introduction}

There is considerable variation in the extent of the association between a blood sucking insect and its host. Blood sucking Diptera such as mosquitoes are temporary ectoparasites, only visiting the host for long enough to obtain a blood meal. Temporary ectoparasites such as tsetse flies, tabanids and mosquitoes tend to take relatively large blood meals, thus limiting the danger from the host by minimising the number of visits paid to it, and acting as an insurance policy in case hosts are difficult to locate in the future. Permanent ectoparasites such as lice are able to take small and frequent blood meals because they spend their whole life on the host, and are therefore not subject to the same feeding pressures or dangers as temporary ectoparasites. Natural selection has ensured that these temporarily ectoparasitic insects have optimised their locomotory systems to maximise the size of meal they can take, while at the same time minimising the risks attendant in carrying it off. This concept is demonstrated in tetse flies (1), and mosquitoes (2), which frequently take a blood meal two to three times their unfed body weight. Such a meal seriously impairs the insect's manoeuvrability and reduces flight speed, which is also affected by the cool ambient temperatures occurring at dawn and dusk when many species of both mosquitoes and tsetse flies normally feed. To compensate, the fed tsetse fly generates heat endogenously by 'buzzing' with its wings, raising the thoracic temperature and enabling it to maximise its lift and flight speed (3). In tsetse flies for example, the blood meal is not only important for reproduction in females, but also for basic metabolic processes, nutrition and flight. In males, at least $50 \%$ of the blood meal is used for flight, compared with only about $25-30 \%$ in females (4).

The difficulty which hungry haematophagous insects have in locating their next blood meal depends upon the closeness of their association with the host. The behaviour patterns involved in locating a host have no strict sequence, thus allowing a flexible response on behalf of the insect to the differing circumstances in which it will encounter hosts. It is probable, however, that insects mostly encounter host-derived stimuli in a particular sequence. The various behaviour patterns involved in host location can be divided into three stages (5).

1. Appetitive search - driven by hunger the insect indulges in non-orientated behaviour likely to bring it into contact with stimuli derived from a potential host. This usually occurs at a specific time of day regulated by the insect's circadian rhythm.

2. Activation and orientation - upon receipt of host stimuli the insect switches from behaviour patterns driven by appetitive searching to oriented host location behaviour. This is driven by host stimuli which are of increasing variety and strength as the insect and host come closer together.

3. Attraction - the final phase. Host-derived stimuli, such as the size, shape and colour of the target, are? used to bring the insect into the host's immediate vicinity and the decision of whether or not to contact the host is made.

\section{Appetitive search}

The delay between emergence and first blood meal may be due to the period of several days required for maturation or the inability of the insect to penetrate the skin until its mouthparts are sufficiently hardened, which takes about 24 hours (6).

As the post emergence delay progresses or as the time since the last blood meal lengthens, the insect becomes increasingly in need of food and is more likely to begin host searching. These patterns are not inviolable; species commonly show variations in their circadian rhythm when collected from different habitats or at different times of the year. As the need for food increases, host searching activity intensifies and occupies increasingly longer periods of time (7). Most of the activity seen in hungry insects is appetitive behaviour, i.e. behaviour that maximises the chances of the insect contacting a signal or derived from a host animal.

The simplest form of appetitive behaviour is to remain $\underset{\mathrm{N}}{\mathrm{N}}$ stationary and wait for a host stimulus to arrive. Provided 
that the insect chooses the resting position carefully, this is a reliable strategy, combining maximum energy conservation with a strong chance of encountering a host. For Diptera and other blood feeding flying insects, this energy conserving strategy is important, as continual active host seeking would be potentially life threatening to the insect. However, for relatively sedentary arthropods such as ticks, their slow metabolic rate means that they can sit and wait for a host for a long period of time without detrimental effect. Flying insects take advantage of this situation by restricting their periods of host seeking to certain times of the day (8), regulated by their circadian rhythm. This strategy therefore optimises their ability to conserve energy but at the time time enables them to find a potential host.

Another strategy is for the insect to fly across a wind stream, blowing from a consistent direction, allowing the maximum number of air streams to be monitored for a particular energy expenditure (9). However, theoretically speaking, if the wind direction veers by more than $30^{\circ}$ from the mean, downwind flight becomes the most energy efficient method of sampling the maximum number of air streams (10).

\section{Activation and Orientation}

Activation occurs when the insect comes into contact with a suitable signal from a potential host animal, which may affect the insect in two ways, either altering the behavioural awareness of the insect to the potential host without causing it to move, or directly causing the insect to switch from endogenously driven appetitive searching to orientated host location behaviour. The insect then uses the information contained in the host-derived signal to orientate towards the host. The detection of very small changes in carbon dioxide levels stimulates the mosquito to switch to active host seeking, but is also used to orientate the insect towards its host.

It is generally accepted that carbon dioxide is involved in both activation and orientation of blood sucking insects. In mosquitoes, it is the change in the concentration of carbon dioxide rather than the level encountered which is the important factor in eliciting a behavioural response (11), small changes causing it to switch to active host searching. In still air carbon dioxide activates the mosquito $(12,13,14,15)$, but there is no orientation to the source. To be fully effective the carbon dioxide must be pulsed or accompanied by other host odours, for example fatty acids and ketones, an example of a dual stimulus.

Dual stimuli cover a spectrum of responses ranging from synergism (where the two stimuli give an overall reaction which is greater than the sum of the two stimuli given separately), to an interactive where one stimulus primes the insect to respond to the second which, if given alone, has no effect $(16,17,18)$. For example, lactic acid is an activating and orientating stimulus to mosquitoes, but only if carbon dioxide is also present in the air stream
$(19,20)$. Not all species, even closely related one respond to the same combinations of stimuli. Thes distinctive responses to combinations of different stimu焉 have developed in insects for a number of reasonse Multiple stimuli are likely to be a more reliable indication of the presence of a host than one stimulus alone, because. a single stimulus may be of non-host origin. Respondin to stimuli received in combination is likely to maximise the chances of a host encounter, while minimising energ consumption.

When searching for an odour plume and/or carbo $\frac{\mathscr{6}}{8}$ dioxide gradient, the insect must fly into the win\& (positive anemotaxis). To be able to, the insect must know in which direction the wind is blowing. Mosquitoes probably orientate themselves upwind by adopting ant undulating flight pattern, determining wind direction b observing the apparent movement of fixed objects in the environment (optomotor anemotaxis) $(21,22)$. Tsetse flies will also use upwind, optomotor anemotaxis as a $\mathrm{R}$ efficient method for locating hosts $(23,24)$. Convectio $\vec{m}$ currents given off by a warm body enable mosquitoes $t \vec{g}$ orientate to a host very effectively over short distances To obtain the optimum benefit from these currents, theyr approach the host flying close to the ground, or slightlo above the top of the prominent vegetation. The ins probably adopts a compromise between, on the one hollon the height giving the most favourable wind speed for 1 is odour detection, optomotor anemotaxis and upwind fligis and on the other, a height permitting stealthy approacto the host while giving maximum protection fronog predators.

Vision is important in the activation and orientation many blood sucking insects. Not surprisingly it is mas widely used by diurnal insects living in open habitats, fo example the biting blackfly Simulium $(25,26,27)$. The distance at which visual orientation becomes importane depends on the visual activity of the insect and the siz $\overrightarrow{\bar{E}}$ and visibility of the object concerned. Theoretically visual information available to the blood sucking insect is obtained from the intensity contrast between the target and the background, and also from colour.

Visual cues play a role in host location, but man mosquitoes are active at night when visibility may be minimal, thus it is likely that vision is integrated wit information from other sources in the search for a hos Typically the insect is initially activated by host odout and uses this to track the host from a distance. As it close로. in on the host, visual information is used in the finas stages of orientation.

\section{Attraction}

Vision, as well as being important in activation an orientation, will play an important role in the attraction of blood sucking insects to the host and will indicate whether and where to land. Insects typically prefer to land on darro rather than light coloured hosts. The insect is able t@ recognise different parts of the host animal and the 
preference varies with species. For example, different tabanid species select markedly different landing sites on cattle (28), mosquitoes tend to have a preference for the extremities of a target $(29,30)$, and simuliids are frequently caught on projections such as the ears (31). Mosquitoes are also activated towards large visible targets but in the absence of any host-derived stimuli or movement, they veer away and will not land (32). It must also be noted that even if all other host-derived stimuli are present and the insect lands, it will only remain momentarily if the surface does not elicit the correct tactile stimulus.

Odour is also important. Generally, those odours which are involved in activation and orientation are also significant in the attraction phase of host location, but as the insect approaches its quarry, new stimuli continually become available, enabling the host to be pinpointed more effectively. One of the most important of these stimuli is heat. Aedes aegypti shows maximal spike frequency changes in the cold and hot receptors of its antennal sensilla coeloconia (olfactory or other sense organs of an insect, consisting of a small projecting cone whose base is connected to a nerve fibre) in response to temperature changes of $+0.2^{\circ} \mathrm{C}(33)$. Little information is available on the distance over which heat is effective, but it has been demonstrated that there are marked convection currents, with local thermal differences of $1^{\circ} \mathrm{C}$ or more, at up to and beyond $40 \mathrm{~cm}$ from a human arm (34). Such a target would be easily thermally detectable by Ae. aegypti from a considerable distance. Anopheles melas has been shown to respond to a single calf at a distance of 14 metres and to two calves at over 36 metres (35). Heat may indicate the presence of a host in various ways. The insect may respond to the radiant heat emitted by the host, to temperature gradients between host and insect (connective heat), or directly to body heat of the host once it has been contacted (conducted heat). However, it is often difficult to establish which heat stimulus is being utilised by the insect. The degree of involvement of water vapour in short range orientation-attraction is unclear, but the upwind movement of the mosquito Ae. aegypti in a stream of warmed air is enhanced if the relative humidity of the air is between 40 and $60 \%$ (18).

Once a suitable host has been located and the insect lands, it probes the surface of the host before it starts to feed. Probing and engorgement occur in response to the quality and quantity of host derived stimuli (36), which may be modulated by the insect's degree of hunger ( 7,37 , $38)$, water deprivation $(39,49)$, previous feeding experience (41), or reproductive state (42). This flexibility of close range responsiveness allows the insect to make the most of the differing circumstances in which it contacts hosts.

External factors affecting the readiness of insects to probe include vibration, surface texture, skin, hair and feather thickness, carbon dioxide levels, odours, visual stimuli, chemical stimuli, heat and moisture levels. Of these, heat is one of the most important (36). In Rhodnius prolixus, heat alone is a sufficient stimulus to initiate a probing response (43), but in mosquitoes (33), it is not always a necessary stimulus (44).

\section{The Blood Meal}

Blood feeding is regulated by a circadian rhythm, with most species of mosquito being nocturnal or crepuscular (active during twilight hours) and some diurnal. Most nocturnal species have a peak biting rate in the early hours of the morning after midnight. Crepuscular species have a concentrated period of biting in the 20 minutes following sunset, but no similar activity period occurs at sunrise. Diurnal species have two peaks of activity, between 6-7 am and before sunset (5-6 pm). Most mosquitoes show gonotropic concordance (the blood meal is largely used to provide resources for the reproductive effort) in which there is a direct link between bloodfeeding and oviposition, so that each blood meal is followed by the maturation and oviposition of a batch of eggs, provided that the quantity of blood ingested exceeds a minimum threshold level. Insemination generally occurs during the period of maturation before the first blood meal.

The size of blood meal is affected by a range of factors including ambient temperature, insect age, mating status, stage of the gonotrophic cycle, previous feeding history and source of the blood meal. Nevertheless, it is fair to say that temporary ectoparasites tend to take very large blood meals in relation to their body size, which are commonly twice their unfed body weight. Taking such a large volume of blood in one meal may be advantageous in several ways. If hosts are difficult to locate, then the insect must make the most of each encounter. It may also be selectively advantageous to take the largest meal possible if the extra time and energy required for more frequent host visits could be spent in reproductive activity. The disadvantages of taking a large blood meal are overcome by making use of the very efficient diuretic properties of the insect gut, which enables the majority of the $80 \%$ water $(45,46)$ contained in the blood meal to be excreted. Most of this excess water is not required by the insect, and its rapid excretion reduces its weight and quickly restores its manoeuvrability. In juvenile bugs, ticks and lice, the ingestion of a large blood meal is vital for continued maturation and development to the next stage moulting.

In adult insects the blood meal is largely used to provide resources for the reproductive effort. However, some species of haematophagus insect are able to produce at least one batch of eggs without the need for a blood meal (autogeny). Some species of mosquito have no need to feed on blood at all. Other mosquitoes as well as some species of sand fly, blackfly and tabanids are autogenous. Autogeny may be facultative or obligate. The flexibility allowed by facultative autogeny is determined by the external cues that the insect receives from its 
environment, which then enables it to establish whether autogeny is the best strategy for the quality of larval feeding and the availability of hosts for the adult. Obligately autogenous insects do not have this alternative. In anautogenous insects, the feeding event is followed by the development and subsequent oviposition of a batch of eggs, providing that the quantity of blood ingested exceeds a minimum threshold level. The remainder of the resources accumulated from the ingestion of the blood meal are apportioned between basal metabolism and locomotion. However, to enable the production of viable eggs, some species of insect must feed from a specific host.

The factors determining host choice may be related to evolutionary patterns, but are usually not clear cut. Typical factors influencing host choice usually act in combination, and include behavioural, geographical, temporal and genetic considerations.

It has been suggested (47), that once the insect has become associated with a narrow range of hosts, specialisations in its physiology will occur which limit the range of other hosts it can exploit. Natural selection will ensure that its systems will become tuned to the exploitation of the resources of its major hosts, which may restrict its ability to deal with unusual situations. In extreme cases, the choice of the wrong host can lead to the death of the insect.

The availability of hosts is often a prime factor in determining host choice. To be available there must be a temporal as well as a geographical overlap between the insect and its host because most temporary ectoparasites only feed during a well-defined period of the day. Seasonal variation in the choice of host has been recognised in some blood sucking insects, and it can have serious consequences for disease transmission to man. In North America, the arbovirus which causes St. Louis encephalitis is transmitted during the summer months by the mosquitoes Culex nigripalpus and C.tarsalis. Both these show marked seasonal changes in their feeding patterns, switching from bird feeding in the winter and spring to mammal feeding in the summer when arbovirus transmission to Man occurs $(48,49,50)$. Insects often choose to feed on particular individuals from among the preferred host species, which may well have implications for disease transmission (51).

The seasonal variation of insect populations may play a part in the seasonal changes in host choice, due to changes in the defensive behaviour of hosts to the different levels of attack (52), which may be important in the transmission of some zoonoses to Man.

The chemical and behavioural relationships between the insect, its host and pathogens, have allowed some species to become effective vectors of disease, and a greater understanding of insect behaviour will reduce the threat which these feeding habits currently pose to Man.

\section{Conclusion}

Insects bite to obtain blood, which is then used in $\bar{\pi}$ variety of ways dependent on the species an $\$$ development stage. In juvenile bugs, ticks and lice, fo example, the ingestion of a large blood meal is vital fo continued maturation and development to the next stage. by moulting. In adult insects the blood meal is largel $\overrightarrow{\mathrm{S}}$ used to provide resources for the reproductive efforto although autogenous species are able to produce at leas one batch of eggs without the need for blood. Each bloo meal is followed by the maturation and oviposition of $\bar{\phi}$ batch of eggs, provided that the quantity of blood ingeste exceeds a minimum threshold level. The remainder of the resources from the meal are apportioned between basa $\Phi$ metabolism and locomotion, with the majority of the water being excreted.

\section{REFERENCES}

1. LANGLEY PA. Post-teneral development of thoracie flight musculature in tsetse flies Glossina austeni an $\$$

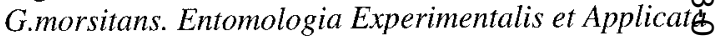
1970; 13: 133-140.

2. NAYAR JK, SAUERMAN DM. The effects of nutrition oㅍ survival and fecundity in Florida mosquitoes. Pats 2 Utilisation of a blood meal for survival. $J$ 16ed Entomol 1975b; 12: 99-103.

3. Howe MA, Lehane MJ. Post-feed buzzing in Ph⿻ tsetse Glossina morsitans morsitans, is ado endothermic mechanism. Physiol Entomol 1986; ํํㅇ 279-286.

4. BuRSELl E, TAYLOR P. An energy budget for Glos (Diptera: Glossinidae). Bull Entomol Res 1980; 得 187-196.

5. SUTCLIFFE JF. Distance orientation of biting flies to their hosts. Insect Science and its Applications 198 8: 611-616.

6. BATES M. The natural history of mosquitoes. Ne쓸 York: Macmillan, 1949.

7. BRADY J. The visual responsiveness of the tsetse f Glossina morsitans (Glossinidae) to moving object the effects of hunger, sex, host odour and stimultọs characteristics. Bull Entomol Res 1972; 62: 257-279.

8. Dethier VG. The Hungry Fly, Cambridge Mas Harvard University Press, 1976.

9. Linsenmair KE; Die Windorientierung Läfende் Insekten. Fortschr Zool 1973; 21: 59-79.

10. SABELIS MW, SCHIPPERS P. Variable wind direction and anemotactic strategies of searching for an odo plume. Oecologica 1984; 63: 225-228.

11. WRIGHT RH, KELLOGG FE, Response of Aedes aegyp to moist convection currents. Nature 1962; 194: 40. 403.

12. OMER SM, Gillies MT. Loss of response to carbon dioxide in palpectomized female mosquitoe Entomologia Experimentalis et Applicata 1971; 14: 251-252. 
13. BuRsEll E. Effects of host odour on the behaviour of tsetse. Insect Science and its Applications 1984; 5: 345-349.

14. BuRSELl $\mathrm{E}$. The effect of wind-borne odours on the direction of flight in tsetse flies Glossina spp. Physiol Entomol 1987; 12: 149-156.

15. WARNES ML, FINLAYSON LH. Responses of the stable fly, Stomoxys calcitrans (L.) (Diptera Muscidae) to carbon dioxide and host odours. I. Activation. Bull Entomol Res 1985; 75: 519-527.

16. LAARMAN JJ. The host-seeking behaviour of anopheline mosquitoes. Trop Georgr Med 1958; 10: 293-305.

17. Bos HJ, LaArman JJ. Guinea pig lysine, cadaverine and oestradiol as attractants for the malaria mosquito Anopheles stephensi. Entomologia Experimentalis et Applicata 1975; 18: 161-172.

18. Bar-Zeev M, Maibach HI, Khan AA. Studies on the attraction of Aedes aegypti (Diptera: Culicidae) to man. J Med Entomol 1977; 14: 113-120.

19. Smith CN, Smith N, Gouck HK. L-Lactic acid as a factor in the attraction of Aedes aegypti to human hosts. Ann Entomol Soc Am 1970; 63: 760-770.

20. Price CD, Smith N, CARLson DA. The attraction of female mosquitos (Anopheles quadrimaculatus Say) to stored human emanations in conjunction with adjusted levels of relative humidity, temperature and carbon dioxide. Journal of Chemical Ecology 1979; 5: 383395.

21. Kennedy JS. The visual responses of flying mosquitoes. Proceedings of the Zoological Society of London. 1940; 109: 221-242.

22. DAVID CT. Mechanisms of directional flight in wind. In: Mechanisms in insect olfaction. TL Payne, MC Birch and CES Kennedy (Eds), Ch.5, pp 49-57. 1986.

23. VAlE GA. New field methods for studying the response of tsetse flies (Diptera: Glossinidae) to hosts. Bull Entomol Res 1974a; 64: 199-208.

24. Colvin J, Brady J, Gibson G. Visually guided, upwind turning behaviour of free-flying tsetse flies in odour-laden wind: a wind-tunnel study. Physiol Entomol 1989; 14: 31-39.

25. FredeEN FJH. A trap for studying the attacking behaviour of blackflies Simulium articum Mall. Canadian Entomologist 1961; 93: 73-78.

26. PEsChKEN DP, ThorsteInSON AJ. Visual orientation of blackflies (Simuliidae Diptera) to colour, shape and movements of targets. Entomologia Experimentalis et Applicata 1965; 8: 282-288.

27. THOMPSON BH. Studies on the attraction of Simulium damnosum s. l. (Diptera: Simuliidae) to its hosts. I. The relative importance of site, exhaled breath and smell. Tropenmed Parasitol 1976; 27: 455-473.

28. Mullens BA, Gerhardt RR. Feeding behaviour of some Tennessee Tabanidae. Environ Entomol 1979; 8: 1047-1051.
29. Fallis AM, Bennett, GF, Griggs G, Allen T. Collecting Simulium venustum female in fan traps and on silhouettes with the aid of carbon dioxide Can $J$ Zool 1967; 45: 1011-1017.

30. BRownE SM, BENNETT GF. Response of mosquitoes (Diptera: Culicidae) to visual stimuli. J Med Entomol 1981; 18: 505-521.

31. WenK P, SCHLORER G. Wirtsorientierung und Kopulation bei blutsaugenden Simuliiden (Diptera). Zoologische Jahrbücher Abteilung für Anatomie und Ontogenie der Tiere 1963; 80: 81-134.

32. Bidlingmayer WL, Hem DG. Mosquito (Diptera: Culicidae) flight behaviour near conspicuous objects. Bull Entomol Res 1979; 69: 691-700.

33. Davis EE, SoKolove PG. Temperature responses of the antennal receptors in the mosquito, Aedes aegypti. J Comp Physiol 1975; 96: 223-236.

34. WRIGHT RH. Tunes to which mosquitoes dance. New Scientist 1968; 37: 694-697.

35. ClEMENTS AN. The biology of mosquitoes, Volume 1, London: Chapman and Hall, 1992.

36. FrIEND WG, SMITH JJB. Factors affecting feeding in blood sucking insects. Annu Rev Entomol 1977; 22: o 309-331.

37. BRADY J. Changes in the probing responsiveness of starving tsetse flies (Glossina morsitans Westw 9 (Diptera: Glossinidae). Bull Entomol Res 1973; 632 웅 247-255.

38. Friend WG, SMITH JJB. Feeding in Rhodnil prolixus; increasing sensivity to ATP during prolonged food deprivation. I Insect Physiol 1975; 210 1081-1084.

39. KHAN AA, MaIBACH HI. A study of the probing response of Aedes aegypti. I. Effect of nutrition on probing. J Econ Entomol 1970; 63: 974-976.

40. KHAN AA, MAIBACH HI. A study of the probing response of Aedes aegypti. II. The effect of dessication $\overrightarrow{\overrightarrow{0}}$ and blood feeding on probing to skin and an artificial target. J Econ Entomol 1971; 64: 439-442.

41. Mitchell BK. Reinouts van HaGa-Kelker HA. A comparison of the feeding behaviour in teneral and post-terenal Glossina morsitans (Diptera: Glossinidae) using an artificial membrane Entomologica Experementalis et Applicata 1976; 20: 105-112.

42. Tobe SS, Davey KG. Volume relationships in the pregnancy cycle of the tsetse fly Glossina austeni. Can J Entomol 1972; 30: 999-1010.

43. Wigglesworth VB, Gillett JD. The function of the antennae in Rhodnius prolixus and the mechanism of orientation to the host. J Exp Biol 1934; 11: 120-139.

44. JONES JC, PILlitT DR. Blood-feeding behaviour of adult Aedes aegypti mosquitoes. Biol Bull 1973; 145: $\frac{\pi}{2}$ 127-139.

45. Albritton A. Standard values in blood. London, ㅇ Saunders, 1952.

46. AltMAN PL, DitTMER DS. Blood and other body N fluids. In: Respiration and circulation. Bethseda, Ma: ত్ 
Federation of American Societies of Experimental Biology, 1971.

47. LeHANE MJ. Biology of blood-sucking insects. London: Harper Collins Academic, 1991.

48. Tempelis CH, Washino RK. Host feeding patterns of Culex tarsalis in the Sacramento Valley, California with notes on other species. J Med Entomol 1967; 4: 315-318.

49. EDMAN JD, TAYLOR DJ. Culex nigripalpus: seasonal shift in the bird-mammal feeding ratio in a mosquito vector of human encephalitis., Science 1968; 161: 6768.
50.EDMAN JD. Most-feeding patterns of Florid疋 mosquitoes. III. Culex (Culex) and Culex (Neoculex). Med Entomol 1974; 11: 95-104.

51. BURKOT TR. Non-random host selection by anopheline mosquitoes. Parasitology Today 1988; 4: 156-162.

52. EDMAN JD, SPIELMAN A. Blood-feeding by vectorsẹ? physiology, ecology, behaviour, and vertebrat defence. In: The arboviruses: epidemiology anf ecology, Vol 1. T.P. Monath, (ed.). CRC Press. 1988. 